\title{
Tailoring Chirp in Spin-Lasers
}

\author{
Guilhem Boeris ${ }^{1,2}$, Jeongsu Lee ${ }^{1}$, Karel Výborný1,3, and Igor Žutić ${ }^{1 *}$ \\ 1 Department of Physics, State University of New York at Buffalo, NY 14260, USA \\ 2 Département de Physique, Ecole Polytechnique, 91128 Palaiseau Cedex, France \\ 3 Institute of Physics ASCR, v.v.i., Cukrovarnická 10, CZ-16253, Praha 6, Czech Republic
}

(Dated: October 30, 2018)

\begin{abstract}
The usefulness of semiconductor lasers is often limited by the undesired frequency modulation, or chirp, a direct consequence of the intensity modulation and carrier dependence of the refractive index in the gain medium. In spin-lasers, realized by injecting, optically or electrically, spin-polarized carriers, we elucidate paths to tailoring chirp. We provide a generalized expression for chirp in spinlasers and introduce modulation schemes that could simultaneously eliminate chirp and enhance the bandwidth, as compared to the conventional (spin-unpolarized) lasers.
\end{abstract}

Many advantages of lasers stem from their modulation response, in which refractive index and optical gain depend on carrier density $n n^{[12]}$ Modulation $\delta n(t)$ thus generates both the intensity (photon density) $\delta S(t)$ and frequency modulation $\delta \nu(t)$ of the emitted light. Such $\nu(t)$, known as chirp, 1 is usually a parasitic effect associated with linewidth broadening, enhanced dispersion, and limiting the high bit-rate in telecommunication systems. ${ }^{3}$ Various approaches have therefore focused on low-chirp modulation: pulse shaping, ${ }^{[3}$ injection locking,, 4 temperature modulation,, 5 and employing quantum dots as the gain region! ${ }^{6}$ In conventional lasers for small signal analysis ${ }^{6}$ (SSA) in which the quantities of interest are decomposed into a steady state and modulated part $X=X_{0}+\delta X(t)$, the chirp is given by ${ }^{1]}$

$$
\delta \nu(t)=\left[\Gamma g_{0} /(4 \pi)\right] \alpha_{0} \delta n(t),
$$

where $\Gamma$ is the optical confinement factor, $g_{0}$ the gain coefficient, and $\alpha_{0}=\left(\partial \hat{n}_{r} / \partial n\right) /\left(\partial \hat{n}_{i} / \partial n\right)$ is the linewidth enhancement factor, 6 expressed in terms of complex refraction index $\hat{n}=\hat{n}_{r}+i \hat{n}_{i}$ in the active region.

In the emerging class of semiconductor lasers, known as spin-lasers, ${ }^{\sqrt[7]{23}}$ with total injection $J=J_{+}-J_{-}$ containing inequivalent spin up/down contributions $\left(J_{+}\right.$, $J_{-}$), we expect additional possibilities for tailoring chirp. $J_{+} \neq J_{-}$is realized using circularly-polarized photoexcitation or electrical injection from a magnetic contact. The polarization of emitted light resolved in two helicities, $S=S^{+}+S^{-}$, can be understood from the optical selection rules ${ }^{24}$ For example, in the quantum well-based spin-lasers with $J$ close to the lasing threshold, recombination of spin-up (spin-down) electrons and heavy holes yields $S^{-}\left(S^{+}\right)$polarized light. Both amplitude modulation (AM) $\delta J(t)$ [see Fig. 11(a)] and polarization modulation $(\mathrm{PM}) \delta P_{J}(t)$, of injection polarization 24 $P_{J}=\left(J_{+}-J_{-}\right) /\left(J_{+}+J_{-}\right)$, can be readily implemented. With PM the emitted light could be modulated even at a fixed $J$ and $n ! \frac{16}{16}$ While Eq. (1) then suggests a chirpfree operation, we show that such a simple reasoning is not always true and suitable generalization for chirp in spin-lasers is required.

Our generalized picture reveals that AM and PM in spin-lasers enable both reduced chirp and enhanced
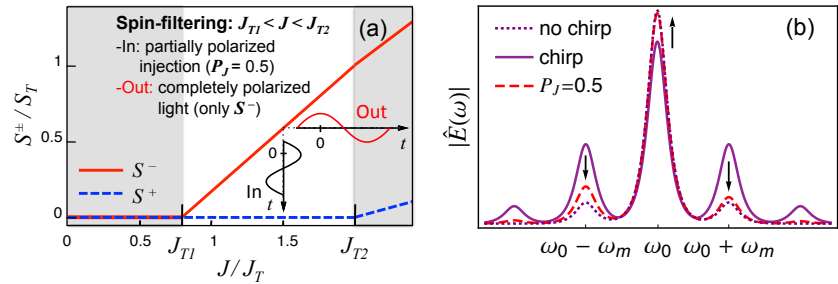

FIG. 1: (Color online) (a) Helicity-resolved photon density $\left(S^{ \pm}\right)$as a function of injection $(J)$, normalized to $S_{T}=S\left(2 J_{T}\right)$ and unpolarized injection threshold $J_{T}$, respectively. For spin-polarized injection, $\left|P_{J}\right|>0$, there are two thresholds ${ }^{16} J_{T 1,2}$ for $S^{\mp}$. AM (harmonic curves) for $J \in\left(J_{T 1}, J_{T 2}\right)$ yields modulation of fully polarized light (spinfiltering, unshaded area). (b) Broadened electric field spectrum for AM. Conventional lasers $\left(P_{J}=0\right)$ without (dotted line) and with chirp (solid), and spin-laser with $P_{J}=0.5$ (dashed) are shown. Arrows indicate the chirp reduction by spin injection. Modulation amplitudes for $P_{J}=0.5$ and $P_{J}=0$ are chosen to provide the same spectra for no chirp. The choice of colors reflects that an unpolarized $S$ is an equal weight superposition of $S^{+}$and $S^{-}$, while for $P_{J}=0.5$ the emitted light is $S^{-}$.

modulation bandwidth, as compared to their spinunpolarized $\left(P_{J}=0\right)$ counterparts. PM could also provide an efficient spin communication. 25

The chirp can be simply quantified by comparing the ratio of the central and first sideband peaks in the emit-

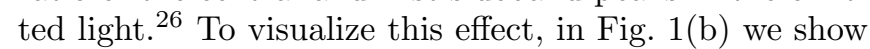
the spectrum of electric field which can be written as ${ }^{2]}$

$$
E(t) \simeq E_{0}\left[1+\delta S(t) /\left(2 S_{0}\right)\right] \operatorname{Re}\left\{e^{i\left[2 \pi \nu_{0} t+\phi(t)\right]}\right\},
$$

where $E_{0}$ is a real amplitude of the field, the phase is $\phi(t)=2 \pi \int_{0}^{t} \delta \nu\left(t^{\prime}\right) d t^{\prime}$, and $\nu_{0}\left(\omega_{0}\right)$ is (angular) frequency of the output light. Using rate equations (REs) we calculate harmonic modulation with $\omega_{m}$ in $\mathrm{SSA}^{27}$ and obtain $\phi(t)=\left[\left|\delta \nu\left(\omega_{m}\right)\right| / \nu_{m}\right] \sin \left(\omega_{m} t+\phi_{\nu}\right)$, where $\phi_{\nu}=\arg \left[\delta \nu\left(\omega_{m}\right)\right]$. The undesirable alteration to the original spectrum caused by chirp can be quantified by the ratio between the heights of the first sidebands with and without chirp. For spin-unpolarized lasers, an indentity ${ }^{2}$ $e^{i \delta \sin x}=\sum_{n=-\infty}^{\infty} J_{n}(\delta) e^{i n x}$, with asymptotic approxi- 
mation $\delta \ll 1$ for Bessel functions $J_{n}(\delta)$, leads to $\frac{113}{13}$

$$
\frac{\text { sideband height with chirp }}{\text { sideband height without chirp }} \simeq \sqrt{1+4\left(\frac{\mathrm{FM}}{\mathrm{IM}}\right)^{2}}
$$

where the ratio of frequency and intensity modulation index FM/IM can be expressed as ${ }^{113}$

$$
\mathrm{FM} / \mathrm{IM}=\left[\delta \nu\left(\omega_{m}\right) / \nu_{m}\right] /\left[\delta S\left(\omega_{m}\right) / S_{0}\right] .
$$

Equation (3) accurately gives the variation of the first sidebands in Fig. 1.(b). The phase induced by the chirp also creates higher order sidebands further away. However, by the spin-polarized injection modulation, chirp and thus alteration of the spectrum can be suppressed.

To define chirp in spin-lasers, we recall that the generalization of the usual model of optical gain term 12116 is $g_{0}\left(n-n_{\text {tran }}\right) \rightarrow g_{0}\left(n_{ \pm}+p_{ \pm}-n_{\text {tran }}\right)=g_{0}\left[(3 / 2) n_{ \pm}+\right.$ $\left.(1 / 2) n_{\mp}-n_{\text {tran }}\right]$, where $g_{0}$ is density-independent coefficient, $n_{\text {tran }}$ the transparency density, and $n_{ \pm}\left(p_{ \pm}\right)$are electron (hole) spin-resolved density. Here 3:1 ratio of $n_{ \pm}$contributions follows from the charge neutrality and the very fast spin relaxation of holes ${ }^{12} p_{ \pm}=n / 2$ and this ratio reflects also the gain anisotropy for $S^{+}$and $S^{-}$.

For spin-lasers the generalization of Eq. (1) is then

$$
\delta \nu(t)=\frac{\Gamma g_{0}}{4 \pi}\left[\frac{3}{2} \alpha_{+} \delta n_{+}(t)+\frac{1}{2} \alpha_{-} \delta n_{-}(t)\right],
$$

where we focus on the spin-filtering regime [Fig. [1(a)], $J \in\left(J_{T 1}, J_{T 2}\right)$, and $\left.\alpha_{ \pm}=\left(\partial \hat{n}_{r} / \partial n_{ \pm}\right) /\left(\partial \hat{n}_{i} / \partial n_{ \pm}\right)\right)^{[28}$ For $P_{J}>0$ the spin filtering implies $S^{-}$emitted light. 29 When $P_{J}=0$ (thus $n_{+}=n_{-}$), Eq. (5) reduces to Eq. (1) since

$$
\alpha_{0}=\left(3 \alpha_{+}+\alpha_{-}\right) / 4 .
$$

While for $P_{J} \neq 0$, Eq. $(6)$ is not always true (since $\alpha_{ \pm}$depends on $n_{ \pm}$), we still use it to relate $\alpha_{ \pm}$and $\alpha_{0}$. This approximation is precise for $J$ slightly below $J_{T 2}=J_{T} /\left(1-P_{J 0}\right)$ where $n_{+}-n_{-} \rightarrow 0$.

With typical spin-lasers, realized as vertical cavity surface emitting lasers, $\frac{8}{11|15| 17}$ in the spin-filtering regime it is accurate to use ${ }^{12}$ vanishing gain compression and spontaneous emission factors $(\epsilon=\beta=0) \stackrel{30}{30}$ With a generalized chirp formulation [Eq. [5] ], we employ REs $\frac{16}{5}$ and SSA to obtain the results from Fig. 1(b). We confirm the chirp suppression in spin-lasers with the spectrum approaching the chirp-free case.

In conventional lasers, the chirp reduction is particularly important for high-frequency modulation where the transient chirp [ $\propto d \ln S(t) / d t$, only weakly $\epsilon$-dependent] is the dominant contribution! ${ }^{1]} \mathrm{FM} / \mathrm{IM}$ is a constant

$$
\frac{\delta \nu\left(\omega_{m}\right) / \nu_{m}}{\delta S\left(\omega_{m}\right) / S_{0}}=-i \frac{\alpha_{0}}{2},
$$

which provides both a suitable way to experimentally extract ${ }^{11}$ the linewidth enhancement factor $\alpha_{0}$, and a
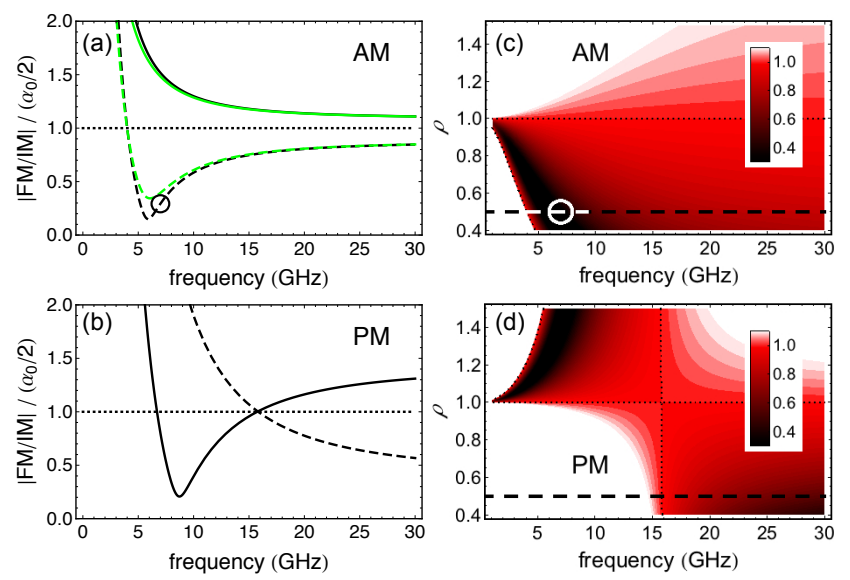

FIG. 2: (Color online) $|\mathrm{FM} / \mathrm{IM}|$ normalized to the conventional value $\alpha_{0} / 2$ for (a) $\mathrm{AM}$ and (b) PM, shown for $\rho \equiv \alpha_{+} / \alpha_{-}=2$ (solid) and $\rho=0.5$ (dashed). Green (gray) curves reveal only a small change for finite electron spin relaxation time ${ }^{27} \tau_{s}=\tau_{r}$. The regime of reduced chirp in spin-lasers (darker regions) is delimited with dotted lines for (c) AM and (d) PM. The circle in (a) and (c) for $\rho=0.5$ represents the sampling point to generate Fig. 1(b). $J_{0}=1.9 J_{T}$ and $P_{J 0}=0.5$ are used in (a)-(d).

simple comparison for chirp in spin-lasers. In the spinfiltering regime $|\mathrm{FM} / \mathrm{IM}|$ depends on the modulation frequency $\omega_{m}$ and the ratio $\rho \equiv \alpha_{+} / \alpha_{-}$[see Eq. (5)]

$$
\left|\frac{\delta \nu / \nu_{m}}{\delta S / S_{0}}\right| / \frac{\alpha_{0}}{2}=\frac{3 \rho \delta n_{+}\left(\omega_{m}\right)+\delta n_{-}\left(\omega_{m}\right)}{3 \delta n_{+}\left(\omega_{m}\right)+\delta n_{-}\left(\omega_{m}\right)}\left(\frac{4}{1+3 \rho}\right) .
$$

$|\mathrm{FM} / \mathrm{IM}|$ of spin lasers is shown in Fig. 2 $\mathrm{A}$ choice of $\rho \in[0.5,2]$ is motivated by our preliminary microscopic calculation (Kubo formalism) of $\alpha_{+}$and $\alpha_{-}$for GaAs. The normalized ratio $|\mathrm{FM} / \mathrm{IM}|<1$ represents the chirp reduction relative to conventional lasers. For AM a change $\rho=2 \rightarrow 0.5$ leads to a smaller chirp for all range of modulation frequencies in Fig. 2(a). Black and gray (green) curves show only a small change in the results for electron spin relaxation time $\tau_{s}$, being infinite and equal to the recombination time $\tau_{r}$, respectively. Since in spin-lasers at $300 \mathrm{~K} \tau_{s} / \tau_{r} \sim 10,15$ it is accurate to choose $\tau_{s} \rightarrow \infty$ in REs for the rest of our analysis.

For PM in Fig. 2(b) the same change $\rho=2 \rightarrow 0.5$ yields a non-monotonic effect on the chirp reduction which, compared to the conventional lasers, is realized at $\nu_{m} \lesssim 16 \mathrm{GHz}(\rho=2)$ and at $\nu_{m} \gtrsim 16 \mathrm{GHz}(\rho=0.5)$, respectively. These trends for AM and PM are further shown in Figs. 2(c) and (d) for a range of $\rho$, where the region of the favorable $|\mathrm{FM} / \mathrm{IM}|$ reduction is delimited with dotted lines. Consistent with Eq. (8), |FM/IM $\mid$ at $\rho=1$ yields the conventional value $\alpha_{0} / 2$, for both AM and PM. Since such a conventional value is retained even for $\mathrm{PM}$ and $\delta n(t)=0$, there is a striking difference between the usual chirp in Eq. (1), and that for spin-lasers in Eq. (5).

Our discussion of FM/IM shows that the chirp is not 

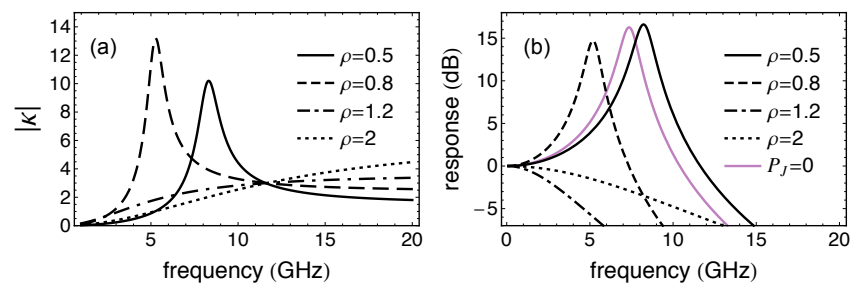

FIG. 3: (Color online) SSA of CM. (a) Chirp-tailoring function $\kappa\left(\nu_{m}\right)$ and (b) modulation response $\left|R\left(\nu_{m}\right) / R(0)\right|^{2}$ are shown for $J_{0}=1.2 J_{T}, P_{J 0}=0.5$ and different $\rho$ 's. The response of conventional laser $\left(\mathrm{AM}, P_{J}=0\right)$ is given (gray/purple) for comparison.

completely removed using PM or AM. However, it is possible to achieve zero-chirp by introducing a scheme we term complex modulation (CM): one of the spin-resolved injections $\left(J_{+}\right.$for $\left.P_{J 0}>0\right)$ is the input signal, while the the other is used only to cancel the chirp. From Eq. (8), the zero-chirp condition is $\delta n_{-}\left(\omega_{m}\right) / \delta n_{+}\left(\omega_{m}\right)=$ $-3 \alpha_{+} / \alpha_{-}=-3 \rho$, which can be satisfied by introducing a chirp-tailoring function $\kappa\left(\omega_{m}\right)$ obtained from SSA

$$
\delta J_{-}\left(\omega_{m}\right)=\kappa\left(\omega_{m}\right) \delta J_{+}\left(\omega_{m}\right) .
$$

Here $\delta J_{+}$is the input modulation responsible for the modulation of emitted light $\delta S^{-}$, while the correction current $\delta J_{-}$compensates the variation of the carrier density to reduce the chirp.

We next use SSA to consider the implications of CM on the modulation bandwidth, shown together with the chirp-tailoring function $\kappa$ in Fig. 3. The CM relaxation oscillation frequency, represented by the peak positions in Figs. 3(a) and (b) for $\rho \leq 1$, can be expressed as

$$
\omega_{R}^{C M} \simeq\left\{\Gamma g_{0} J_{T}\left(J / J_{T 1}-1\right)(1-\rho)\right\}^{1 / 2},
$$

where $J_{T 1}=J_{T} /\left(1+P_{J 0} / 2\right)$ is the reduced threshold in a spin-laser. ${ }^{12}$ The peak positions coincide for $\left|\kappa\left(\omega_{m}\right)\right|$ and for the modulation response function $\frac{16}{16} R\left(\omega_{m}\right)=$ $\left|\delta S^{-}\left(\omega_{m}\right) / \delta J_{+}\left(\omega_{m}\right)\right|$ because the character of $J_{-}\left(\omega_{m}\right) \propto$ $\kappa\left(\omega_{m}\right)$ propagates through $n_{ \pm}$and $S^{-}$into $R\left(\omega_{m}\right)$. For $\rho>1,\left|\kappa\left(\omega_{m}\right)\right|$ increases monotonically with $\omega_{m}$ showing no peak. Zero-chirp is not feasible for $\rho=1$ since it is the same FM/IM as in conventional lasers [Eq. [8]].

By comparing $\omega_{R}^{C M}$ in Eq. 10 for $\rho \leq 1$ to $\omega_{R}^{A M}$ and $\omega_{R}^{P M}$ from Ref. 16; $\omega_{R}^{A M, P M} \simeq\left\{\Gamma g_{0} J_{T}\left(J / J_{T 1}-1\right)\right\}^{1 / 2}$, we see that CM has narrower bandwidth than AM and PM (estimated by $\omega_{R}$ ), for the same $P_{J 0}$. While CM provides a path for removing chirp, it may come at the cost of a reduced bandwidth. However, an optimized value of $\rho=0.5$ in Fig. 3(b) yields simultaneously zero chirp and bandwidth enhancement, as compared to conventional lasers.

What about experimental feasibility to control chirp in spin-lasers? While CM has yet to be attempted, it can be viewed as a combination of AM and PM which individually already lead to an improved chirp (Figs. 1 and 2 and have been demonstrated in spin-lasers. Slow PM has been realized $\sqrt{8}$ using a Soleil-Babinet polarization retarder at a fixed $J \in\left(J_{T 1}, J_{T 2}\right)$. Fast $\mathrm{PM}\left(\nu_{m} \sim 40\right.$ $\mathrm{GHz}$ ) can be implemented with a coherent electron spin precession in a transverse magnetic field ${ }^{[7}$ or a mode conversion in an electro-optic modulator ${ }^{31}$ Recent advances in using birefringence for $\mathrm{PM}^{32}$ suggest that chirp reduction in spin-lasers could be feasible at higher injection, beyond the spin-filtering regime we have considered.

To further enhance the opportunities in spin-lasers, it would be helpful to utilize other gain media and achieve technologically important emission at 1.3 and $1.55 \mu \mathrm{m}$. We expect that our proposals will stimulate additional work towards understanding the spindependence of refractive index (already used for fast alloptical switching ${ }^{33}$ ) and its implications for spin-lasers.

We thank H. Dery, R. Oszwałdowski, A. Petrou, and N. Tesařová for discussions. This work was supported by the NSF-ECCS, AFOSR-DCT, U.S. ONR, NSF-NRI NEB 2020, and SRC.
* Electronic address: zigor@buffalo.edu

${ }^{1}$ L. A. Coldren and S. W. Corzine, Diode Lasers and Photonic Integrated Circuits, (Wiley, New York, 1995).

2 A. Yariv, Optical Electronics in Modern Communications, $5^{\text {th }}$ Edition (Oxford University, New Yok, 1997).

${ }^{3}$ K. Petermann Laser Diode Modulation and Noise, (Kluwer Academic, Dordrecht, 1988).

4 G. H. M. van Tartwijk and G. P. Agrawal, Prog. Quantum Electron. 22, 43 (1998).

5 V. B. Gorfinkel and S. Luryi, Appl. Phys. Lett. 62, 2923 (1993).

6 S. L. Chuang, Physics of Optoelectronic Devices, 2nd ed. (Wiley, New York, 2009).

7 S. Hallstein, J. D. Berger, M. Hilpert, H. C. Schneider, W. W. Rühle, F. Jahnke, S. W. Koch, H. M. Gibbs, G. Khitrova, and M. Oestreich, Phys. Rev. B 56, R7076
(1997).

8 J. Rudolph, D. Hägele, H. M. Gibbs, G. Khitrova, and M. Oestreich, Appl. Phys. Lett. 82, 4516 (2003).

9 J. Rudolph, S. Döhrmann, D. Hägele, M. Oestreich, and W. Stolz Appl. Phys. Lett. 87, 241117 (2005).

10 M. Holub, J. Shin, D. Saha, and P. Bhattacharya, Phys. Rev. Lett. 98, 146603 (2007).

11 S. Hövel, A. Bischoff, N. C. Gerhardt, M. R. Hofmann, T. Ackemann, A. Kroner, and R. Michalzik, Appl. Phys. Lett. 92, 041118 (2008).

12 C. Gøthgen, R. Oszwałdowski, A. Petrou, and I. Žutić, Appl. Phys. Lett. 93, 042513 (2008).

13 I. Vurgaftman, M. Holub, B. T. Jonker, and J. R. Mayer, Appl. Phys. Lett. 93, 031102 (2008).

14 D. Basu, D. Saha, C. C. Wu, M. Holub, Z. Mi, and P. Bhattacharya, Appl. Phys. Lett. 92, 091119 (2008). 
${ }^{15}$ H. Fujino, S. Koh, S. Iba, T. Fujimoto, and H. Kawaguchi, Appl. Phys. Lett. 94, 131108 (2009).

16 J. Lee, W. Falls, R. Oszwałdowski, and I. Žutić, Appl. Phys. Lett. 97, 041116 (2010).

17 D. Saha, D. Basu, and P. Bhattacharya, Phys. Rev. B 82, 205309 (2010).

18 I. Žutić, R. Oszwałdowski, J. Lee, and C. Gøthgen in Handbook of Spin Transport and Magnetism, edited by E. Y. Tsymbal and I. Žutić (CRC Press, New York, 2011).

19 S. Iba, S. Koh, K. Ikeda, and H. Kawaguchi, Appl. Phys. Lett. 98, 08113 (2011).

${ }^{20}$ R. Al-Seyab, D. Alexandropoulos, I. D. Henning, and M. H. Adams, IEEE Photon. J. 3, 799 (2011); M. San Miguel, Q. Feng, and J. V. Moloney, Phys. Rev. A 52, 1728 (1995).

${ }^{21}$ M. Holub and B. T. Jonker, Phys. Rev. B 83, 125309 (2011).

22 D. Banerjee, R. Adari, M. Murthy, P. Suggisetti, S. Ganguly, and D. Saha, J. Appl. Phys. 109, 07 C317 (2011).

23 J. Lee, R. Oszwałdowski, C. Gøthgen, and I. Žutić, Phys. Rev. B 85, 045314 (2012).

${ }^{24}$ I. Žutić, J. Fabian, and S. Das Sarma, Rev. Mod. Phys. 76, 323 (2004); J. Fabian, A. Matos-Abiague, C. Ertler, P. Stano, and I. Žutić, Acta Phys. Slov. 57, 565 (2007).

${ }^{25}$ H. Dery, Y. Song, P. Li, and I. Žutić, Appl. Phys. Lett. 99, 082502 (2011).

26 Y. Arakawa and A. Yariv, Appl. Phys. Lett. 47, 905 (1985).

27 We base SSA on the rate equations Eqs. (4)-(6) for con- ventional lasers and Eqs. (22) and (A1) for spin lasers in Ref. 23. The parameters used for numerical calculations are given in Table I from Ref. 16

28 We can infer that $\alpha_{+} \neq \alpha_{-}$, since the Faraday angle, representing the asymmetry of the refractive indices for $S^{ \pm}$, depends on $n$ [S. Crooker, D. Awschalom, J. Baumberg, F. Flack, and N. Samarth, Phys. Rev. B 56, 7574 (1997); R. Bratschitsch, Z. Chen, and S. T. Cundiff, Phys. Stat. Sol. (c) 0, 1506 (2003)].

29 If there is an emitted light with the other helicity, the chirp from $S^{+}$signal, $\delta \nu^{\prime}(t)$, can be written analogously: $\delta \nu^{\prime}(t)=\Gamma g_{0} /(4 \pi)\left[(1 / 2) \alpha_{+}^{\prime} \delta n_{+}(t)+(3 / 2) \alpha_{-}^{\prime} \delta n_{-}(t)\right]$.

30 Our approximation $\beta=0$ throughout the paper, used also in Fig. 1. accurately captures the expected behavior for experiments on spin-lasers at $300 \mathrm{~K}$, parametrized with $\beta \sim 10^{-5}$ in Ref. 9

31 J. D. Bull, N. A. F. Jaeger, H. Kato, M. Fairburn, A. Reid, and P. Ghanipour, Photonics North 2004: Optical Components and Devices, Ottawa, Canada, [J. C. Armitage, S. Fafard, R. A. Lessard, and G. A. Lampropoulos, Proc. SPIE 5577, 133 (2004)].

32 N. C. Gerhardt, M. Y. Li, H. Jähme, H. Höpfner, T. Ackemann, and M. R. Hofmann, Appl. Phys. Lett. 99, 151107 (2011).

33 Y. Nishikawa, A. Tackeuchi, S. Nakamura, S. Muto, and N. Yokoyama Appl. Phys. Lett. 66, 839 (1995). 